\title{
A HOSPITALIDADE ÀS PESSOAS COM DEFICIÊNCIA OU MOBILIDADE REDUZIDA NOS MEIOS DE HOSPEDAGEM DE LIMOEIRO DO NORTE-CEARÁ
}

\author{
Victor Hugo Santos de Castro ${ }^{1}$, Fernanda Patrícia Sousa Santos ${ }^{1}$ \\ ${ }^{1}$ Instituto Federal de Educação, Ciência e Tecnologia do Ceará - IFCE \\ <vsantosdecastro@yahoo.com.br><nandapssantos@gmail.com> \\ DOI: $10.21439 /$ conexoes.v13i3.1250
}

\begin{abstract}
Resumo. Este estudo aborda a acessibilidade no âmbito hoteleiro. O objetivo geral deste estudo foi analisar a hospitalidade dispensada às pessoas com deficiência ou mobilidade reduzida nos meios de hospedagem de Limoeiro do Norte-Ceará. Os objetivos específicos foram: averiguar se os meios de hospedagem possuem acessibilidade para receber hóspedes com deficiência ou mobilidade reduzida e se esta pode ser um diferencial nos empreendimentos; identificar a demanda deste público nos meios de hospedagem; reconhecer possíveis melhorias no atendimento destes negócios para pessoas com deficiência ou mobilidade reduzida; verificar se os gestores possuem ciência da legislação sobre acessibilidade vigente no país. Quanto à metodologia, tratou-se de uma pesquisa de campo, de caráter exploratório, com abordagem qualitativa. Os dados foram coletados através de entrevistas semiestruturadas. A entrevista foi realizada em dois meios de hospedagem da cidade de Limoeiro do Norte-Ceará. Os resultados apontam que a acessibilidade nos meios de hospedagem é incipiente, e que a hospitalidade no atendimento está relacionada à infraestrutura, a demanda de pessoas com deficiência ou mobilidade reduzida pelos meios de hospedagem de Limoeiro do Norte-Ceará é baixa, e os gestores têm ciência da legislação sobre acessibilidade existente no país, mas superficialmente. A pesquisa conclui que os empreendimentos hoteleiros da cidade em questão, ainda estão caminhando para uma hotelaria mais acessível.
\end{abstract}

Palavras-chaves: Acessibilidade. Hotelaria. Hospitalidade.

\begin{abstract}
This study deals with accessibility in the hotel sector. The overall objective of this study was to analyze the hospitality provided to people with disabilities or reduced mobility in the lodging facilities of Limoeiro do Norte-Ceará. The specific objectives were: to verify if the means of lodging are accessible to receive guests with disabilities or reduced mobility and if this can be a differential in the enterprises; identify the demand of this public in the means of hosting; recognize possible improvements in serving these businesses for people with disabilities or reduced mobility; to verify if managers are aware of the accessibility legislation in force in the country. As for the methodology, it was a field research, exploratory in nature, with a qualitative approach. Data were collected through semi-structured interviews. The interview was carried out in two lodging facilities in the city of Limoeiro do Norte-Ceará. The results indicate that the accessibility in the means of lodging is incipient, and that the hospitality in the service is related to the infrastructure, the demand of people with disabilities or reduced mobility by means of lodging of Limoeiro do Norte-Ceará is low, and the managers have accessibility legislation in the country, but superficially. The research concludes that the hotel developments in the city in question are still moving towards a more affordable hotel.
\end{abstract}

Keywords: Accessibility. Hospitality. Hospitality 


\section{INTRODUÇÃO}

A acessibilidade é um tema relevante, pois apesar da evolução tecnológica, tão presente na contemporaneidade, ainda existem obstáculos a serem superados no cotidiano de pessoas que possuem alguma deficiência ou mobilidade reduzida. As barreiras não são somente as físicas, mas também atitudinais.

Apesar dos embasamentos teóricos existentes que norteiam os direitos deste público, como a Lei 13.146/2015, também denominada Lei Brasileira de Inclusão da Pessoa com Deficiência (Estatuto da Pessoa com Deficiência) e a NBR 9050 (normativa referente à acessibilidade), todos os dias estas pessoas encontram entraves a serem superados, inclusive nos empreendimentos hoteleiros.

Muitos meios de hospedagem não oferecem serviços adequados às necessidades da pessoa com deficiência ou com mobilidade reduzida, a negligência dos empreendimentos pode estar relacionada também à omissão do poder público no que tange à fiscalização.

A hotelaria deve buscar a satisfação plena dos clientes, sobretudo daqueles com deficiência ou mobilidade reduzida. Estes devem ser acolhidos com cordialidade, atenção, presteza, conforto e segurança nos serviços ofertados, sendo estas ações características da boa hospitalidade. Logo, os meios de hospedagem devem estar preparados para atender deficientes auditivos, visuais, motores, obesos, idosos, gestantes e outros.

Objetivando a satisfação do hóspede com deficiência ou mobilidade reduzida, a acessibilidade na hotelaria, aos poucos, busca adaptar seus serviços de recepção, acomodação, restaurante, café, áreas sociais e de entretenimento para a inclusão deste público, que contribui com a atividade turística.

O município de Limoeiro do Norte, localizado no Estado do Ceará, tem crescido no âmbito hoteleiro, uma vez que há demanda de turistas na região. Sendo assim, os empreendedores dos meios de hospedagem devem atentar aos critérios de uma boa hospitalidade, sobretudo no que tange à acessibilidade. Assim, o problema deste estudo foi: como os meios de hospedagem de Limoeiro do Norte-Ceará atendem à demanda de pessoas com deficiência física e mobilidade reduzida?

O objetivo geral deste estudo foi analisar a hospitalidade dispensada às pessoas com deficiência ou mobilidade reduzida nos meios de hospedagem de Limoeiro do Norte-Ceará. Os objetivos específicos foram: averiguar se os meios de hospedagem possuem acessibilidade para receber hóspedes com deficiência ou mobilidade reduzida e se esta pode ser um diferencial nos empreendimentos; identificar a demanda deste público nos meios de hospedagem; reconhecer possíveis melhorias no atendimento destes negócios para pessoas com deficiência ou mobilidade reduzida; verificar se os gestores possuem ciência da legislação sobre acessibilidade vigente no país.

Quanto à metodologia, tratou-se de uma pesquisa de campo, de caráter exploratório, com abordagem qualitativa. Os dados foram coletados através de entrevistas semiestruturadas. A entrevista foi realizada em dois meios de hospedagem da cidade de Limoeiro do NorteCeará. A análise dos dados foi realizada através da transcrição das falas dos entrevistados.

Este estudo é relevante para as pessoas com deficiência ou mobilidade reduzida, para os meios de hospedagem, a fim de que os mesmos se adaptem às exigências do público em questão e possam atender à legislação brasileira vigente. Esta pesquisa também é importante para os profissionais e estudantes da área de ciência sociais aplicadas.

Esta pesquisa apresenta seções sobre hospitalidade e acessibilidade nos meios de hospedagem; a metodologia para a obtenção dos dados; a discussão dos resultados obtidos; e as considerações sobre o estudo.

\section{FUNDAMENTAÇÃO}

\subsection{Acessibilidade e Inclusão}

A atividade turística é um bem comum, de relevância social e econômica, que deve ser estruturada para todos os cidadãos, portanto, deve ser inclusiva. Contudo, o turismo na atualidade, ainda é pouco acessível, principalmente para as pessoas que apresentam algum tipo de deficiência, seja ela intelectual, motora, visual ou auditiva.

Neste contexto, faz-se necessário entender o conceito de acessibilidade, que segundo Cardoso e Strassburger (2012), pode ser entendido como o direito que pessoas com deficiência ou mobilidade reduzida tem de usufruir do uso de ambientes mobiliados, equipamentos, edifícios, aparelhos e meios de comunicação e informação, serviços de transporte, com segurança e autonomia total ou assistida.

De acordo com o Ministério do Turismo, a acessibilidade é requisito que potencializa positivamente diversos aspectos na vida do ser humano, e que deve se fazer presente, independente das circunstâncias (MTUR, 2013).

Oliveira (2012, p. 4) aponta que a "deficiência é um tema de direitos humanos e como tal obedece ao princípio de que todo ser humano tem direito de desfrutar de todas as condições necessárias para o desenvolvimento 
de seus talentos e aspirações, sem ser submetido a qualquer tipo de discriminação".

De acordo com a Organização das Nações Unidas (ONU) na Convenção dos Direitos das Pessoas com Deficiências, "pessoas com deficiência, são aquelas que têm impedimentos de longo prazo de natureza física, mental, intelectual ou sensorial" (BRASIL, 2009a p. 26).

Ainda de acordo com a Convenção dos Direitos das Pessoas com Deficiências, nos termos do decreto n 6949 , em seu artigo $3^{\circ}$, estatui que a pessoa com deficiência tem garantia de igualdade nas oportunidades, direito a não discriminação, à escolha, à acessibilidade, à autonomia e à dignidade (BRASIL, 2009b).

Já as pessoas com mobilidade reduzida possuem limitada capacidade de se relacionar com o meio e de utilizá-lo. Essa limitação pode ser permanente ou temporária. Entende-se, pessoa com mobilidade reduzida o indivíduo com algum tipo de deficiência, idade avançada, obesidade ou gravidez, entre outras características (ABNT, 2015).

Sassaki (2006) afirma que o vocábulo acessibilidade passou a ser usado recentemente, e que se refere ao acesso de pessoas com deficiência a serviços de qualquer natureza. Ainda segundo o mesmo autor, a inclusão se dá "a partir de práticas baseadas na valorização da diversidade humana, no respeito pelas diferenças individuais que se abrem caminhos para a construção de uma sociedade verdadeiramente para todos" (SASSAKI, 2006, p. 20).

As pessoas que possuem uma deficiência ou mobilidade reduzida, devem ter seus direitos assegurados, mas nem sempre estes cidadãos conseguem que seus direitos sejam efetivados, por existirem determinadas imposições, que configuram como desvantagens e obstáculos para este público, como pode ser observado a seguir.

\footnotetext{
As pessoas com deficiência têm os mesmos direitos que as pessoas sem deficiência. No entanto, diferente desse segundo tipo, elas nem sempre conseguem exigir seus direitos com as mesmas condições em que o fazem as pessoas sem deficiência, devido a desvantagens impostas pela restrição de funcionalidades e pela sociedade, que lhes impõe barreiras físicas, legais e de atitude. Essas barreiras são responsáveis pelo distanciamento que existe na realização dos direitos das pessoas com e sem deficiência (OLIVEIRA, 2012. p. 16)
}

Conforme Silva (2004), inúmeras pessoas com deficiência ou mobilidade reduzida não tem assegurado o direito de ir e vir. Estas têm dificuldades de participação social, e de alguma maneira não conseguem se beneficiar dos bens e espaços coletivos pensados e planejados para os ditos "normais", fruto de uma sociedade pensada sob a perspectiva do homem sem deficiência. Em meios de hospedagem esta prática não é diferente.

As pessoas com deficiência ou mobilidade reduzida podem ser consideradas como um novo nicho de mercado que está sendo descoberto pelos profissionais da hotelaria e do turismo, porém trata-se de uma área que precisa ser estudada e aprimorada, com o propósito de identificar as expectativas, interesses e anseios do público e incluí-los (CARDOSO; STRASSBURGER. 2012).

Nesta perspectiva de inclusão, Sassaki (2006) defende a proposta do Desenho Universal. Este pode ser entendido como um ambiente totalmente equipado e confortável para todos os tipos de indivíduos, sem restrições, ou seja, todas as pessoas poderão usufruir igualitariamente dos espaços em comum. O ambiente não é adaptado somente para pessoas com deficiência ou mobilidade reduzida, mas para todo tipo de público.

\footnotetext{
Os produtos universais acomodam uma escala larga de preferências e de habilidades individuais ou sensoriais dos usuários. Qualquer ambiente ou produto deverá ser alcançado, manipulado e usado. Independentemente do tamanho do corpo do indivíduo, sua postura ou sua mobilidade. Dessa forma entende-se que o Desenho Universal não é uma tecnologia direcionada apenas aos que dele necessitam, mas é desenhado para todos os indivíduos, apresentem eles algum prejuízo de mobilidade ou não. A sua ideia é evitar a necessidade de ambientes e produtos especiais, assegurando que todos possam utilizar com segurança e autonomia os diversos objetos e espaços construídos (CARLETTO; CAMBIAGHI 2008. p. 8).
}

O objetivo do Desenho Universal é evitar a criação de ambientes e produtos destinados exclusivamente para algum tipo de público específico. Esse projeto almeja uma abrangência global, pois seu conceito visa atingir o maior número possível de pessoas.

\subsection{Acessibilidade nos Meios de Hospedagem, uma forma de Hospitalidade}

Garantir a inclusão e a acessibilidade nos meios de hospedagem é uma forma de hospitalidade. Os empreendimentos hoteleiros, devem adaptar seus ambientes para garantir o atendimento aos mais diversos públicos. Segundo Stoll (2006), a hospitalidade está relacionada à recepção; ao tratamento cortês; à gentileza.

Acessibilidade nos meios de hospedagem é sinônimo de um ambiente agradável e acolhedor às pessoas com deficiência ou mobilidade reduzida, garantindo satisfação plena das suas necessidades. Ações 
simples como disposições dos móveis, portas mais largas, banheiro adaptado, rampas, elevadores e balcão rebaixado, seriam intervenções facilitadoras para o acesso deste público ao empreendimento.

Essas especificações referentes à acessibilidade são de competência da Associação Brasileira de Normas Técnicas (ABNT), cuja missão é instituir e publicar normas brasileiras desde a década de 40. Portanto, compete a essa entidade, criar as regras de acessibilidade para facilitar e assegurar livre acesso aos indivíduos com deficiência ou mobilidade reduzida.

Cardoso e Strassburger (2012) reconhecem que não existe no país nenhuma norma que regulamente o uso do símbolo internacional de acessibilidade, de modo a identificar os meios de hospedagem que possuem adaptações adequadas a este público. No Sistema Brasileiro de Classificação dos Meios de Hospedagem (SBClass), o termo acessibilidade não tem muita relevância para a obtenção das estrelas, contudo, não é raro encontrar meios de hospedagem com cinco estrelas, cujo os espaços não são adequados às necessidades dos clientes com deficiência ou mobilidade reduzida.

Oliveira (2012) reitera que de acordo com o censo de 2010 do Instituto Brasileiro de Geografia e Estatística (IBGE), 45,6 milhões de brasileiros possuem alguma deficiência, equivalendo a $23,9 \%$ da população. Destes, $7 \%$ possuem deficiência no aspecto motor, $5,1 \%$ problemas auditivos e $18,8 \%$ apresentam alguma dificuldade visual.

Ainda de acordo com a mesma autora, a região nordeste apresentou a maior concentração de pessoas com deficiência, equivalendo a $26,63 \%$ da população. Segmentando por deficiência, os percentuais são: $21,2 \%$ possuem problemas visuais, 5,8\% auditivos, 7,8\% motores e $1,6 \%$ mentais ou intelectuais.

Segundo Sansiveiro e Dias (2005), existem seis barreiras encontradas por pessoas com deficiência ou mobilidade reduzida em locais de atividades turísticas, a saber: arquitetônicas, de atitude, de comunicação, de métodos, de instrumentos e programáticas. A ação prioritária é eliminar os aspectos de natureza arquitetônica, com a remoção dessas barreiras, a sociedade conviverá mais ativamente com esta parcela da população que geralmente encontra-se excluída de atividades sociais como o lazer e o turismo, como pode ser observado abaixo.

Possuir algum tipo de deficiência em um país marcado com grandes contrastes e desigualdades sociais, como o Brasil, significa estar mais distante de receber atendimento de qualidade, uma educação de bom nível, e uma digna oportunidade de trabalho no mercado formal. Um grande desafio com inúmeros obstáculos tem que ser en- frentados ainda pelo deficiente, quando se busca atividades relacionadas ao lazer e ao turismo (SANSIVEIRO; DIAS 2005 p. 4)

Vale ressaltar que a ausência de acessibilidade no cenário atual dificulta o acesso das pessoas com deficiência ou mobilidade reduzida à educação, ocasionando falta de qualificação e, por conseguinte, oportunidades, destas ingressarem no mercado de trabalho, fator relevante para a inclusão social e possível qualidade de vida (SANSIVEIRO; DIAS, 2005).

Lashley (2004, p. 4) afirma que o meio de hospedagem para receber bem um hóspede com deficiência ou mobilidade reduzida deve " além dos itens básicos de hospedagem, o hoteleiro tem o dever de proporcionar bem-estar a esse hóspede, oferecendo adequadas condições e estruturas físicas para que possa garantir a comodidade do mesmo". Esta colocação reafirma a importância da acessibilidade nos meios de hospedagem e atendimento dos anseios dos referido público.

\section{METODOLOGIA}

Tratou-se de um estudo de campo, de caráter exploratório, com abordagem qualitativa. Para Aguiar (2011), a pesquisa qualitativa tem como objetivo analisar as características objetivas e subjetivas, decorrentes de observações, atitudes e opiniões, sem muita ênfase para aspectos numéricos. Segundo Silva (2015), a pesquisa qualitativa traduz um universo de significados, motivações, crenças, aspirações, atitudes e valores.

O cenário da pesquisa foram dois meios de hospedagem de Limoeiro do Norte-Ceará, de um universo de nove empreendimentos. O critério para a escolha dos meios de hospedagem foi a boa repercussão nas redes sociais. Os nomes das instituições foram preservados. A coleta de dados foi realizada através de entrevistas semiestruturadas, agendadas com antecedência e de acordo com a disponibilidade dos gestores dos meios de hospedagem, que foram os participantes deste estudo.

A entrevista foi aplicada em junho de 2016 e foi composta por cinco perguntas abertas para que os entrevistados pudessem discorrer. As entrevistas foram gravadas, para posterior análise dos dados, que foi realizada através da transcrição das falas dos participantes.

\section{RESULTADOS E DISCUSSÕES}

A primeira pergunta da entrevista versou sobre a acessibilidade nos meios de hospedagem, os resultados apontam que as adaptações nos empreendimentos hoteleiros de Limoeiro do Norte-Ceará são mínimas ou 
inexistentes, podendo causar desconforto e constrangimento ao hóspede com deficiência ou mobilidade reduzida no período de permanência, como pode ser observado nas falas abaixo dos entrevistados.

\begin{abstract}
Nós temos apartamentos adaptados para pessoas com deficiência. Os quartos adaptados estão no térreo, porque não temos elevador, somente escadas. Ao todo, são três unidades habitacionais acessíveis, uma delas exclusiva para cadeirante e as outras para pessoas com mobilidade reduzida, como idosos. O restante do empreendimento não possui adaptações, pois quando inauguramos, não haviam tantas exigências quanto na atualidade, não houve um planejamento para todas as variáveis envolvidas na receptividade de pessoas com deficiência [...] Depois do negócio já construído e em funcionamento fica mais complicado para fazer adaptações (ENTREVISTADO 1, PESQUISA DIRETA, 2016).
\end{abstract}

Através da colocação, observa-se que a acessibilidade do meio de hospedagem limita-se ao apartamento, excluindo as áreas de lazer, recepção, restaurante, por exemplo. Nesse caso faz-se necessário uma maior conscientização dos empreendedores do ramo hoteleiro na cidade de Limoeiro do Norte-Ceará, no que diz respeito ao conceito de acessibilidade. Ressalta-se que não há complicações em adaptar a infraestrutura de um negócio às necessidades de um cliente com deficiência ou mobilidade reduzida, como foi mencionado.

Corroborando com o exposto, Cardoso e Strassburger (2012) apontam que muitos dos gestores compreendem que as adaptações nos meios de hospedagem geram gastos, que para muitos deles são desnecessários. Porém, algumas modificações ambientais são de suma importância e contribuiriam para uma boa permanência de pessoas com deficiência ou mobilidade reduzida no empreendimento.

"No momento não ofertamos apartamentos para pessoas especiais, o empreendimento tem um projeto futuro para fazer adaptações para este público" (ENTREVISTADO 2, PESQUISA DIRETA, 2016).

O primeiro passo para a mudança, é aproximar-se da temática. Erroneamente, muitas pessoas utilizam a expressão "pessoas especiais", é essencial utilizar a nomenclatura correta para este público que é pessoa com deficiência ou pessoa com mobilidade reduzida. Observa-se ainda que a inquietação referente à acessibilidade ainda se limita à teoria, sendo que a hospitalidade requer ações práticas para bem acolher, portanto, tornase urgente intervenções inclusivas no empreendimento em questão.

O segundo questionamento referiu-se a demanda de pessoas com deficiência ou mobilidade reduzida pelos serviços dos meios de hospedagem. Observou-se que a procura por apartamentos deste público específico é baixa, como pode ser averiguado nas colocações dos entrevistados, a seguir.

"A maioria das vezes os apartamentos são ocupados por pessoas sem deficiência, a frequência deste público é pequena, mas temos apartamentos para recebê-los" (ENTREVISTADO 1, PESQUISA DIRETA, 2016). “A maioria dos clientes já sabe que não temos apartamentos adaptados, então é muito difícil a procura. Durante todo o ano, tivemos duas ou três pessoas que ligaram para perguntar se recebíamos pessoas com necessidades especiais" (ENTREVISTADO 2, PESQUISA DIRETA, 2016).

Corroborando com as falas dos entrevistados, Cardoso e Strassburger (2012) afirmam que na hotelaria a acessibilidade ainda é realizada em baixa escala, geralmente são atendidas, somente, suas necessidades mais básicas. A legislação estabelece que $5 \%$ das unidades habitacionais dos meios de hospedagem estejam adaptadas para receber pessoas com deficiência ou mobilidade reduzida, e que se localizem em áreas de fácil acesso.

A terceira interrogação abordou a acessibilidade como diferencial no âmbito hoteleiro. Foi constatado que os meios de hospedagem ainda se utilizam desta obrigação, como uma estratégia de marketing, como pode ser observado na fala do entrevistado, abaixo.

“Com certeza é um diferencial, porque este público também viaja, tem emprego, e eles precisam de hospedagem quando se deslocam, então, o setor hoteleiro deve atender esta demanda que cresce cada vez mais" (ENTREVISTADO 2, PESQUISA DIRETA, 2016).

A acessibilidade nos meios de hospedagem, não pode ser vista apenas na perspectiva mercadológica, não foi mencionado que as adaptações para as pessoas com deficiência ou mobilidade reduzida são necessárias por questões humanitárias, afinal, a hotelaria lida com o bem receber, e a hospitalidade deve abranger os mais diversos públicos, primando assim, pela inclusão.

A quarta inquirição versou sobre como poderiam ser realizadas melhorias no atendimento ao hóspede com deficiência ou mobilidade reduzida. Foi identificado que os meios de hospedagem desconhecem as adaptações ambientais e atitudinais mais adequadas ao referido público, como pode ser observado nas colocações abaixo.

"Não sei exatamente o que poderia ser feito" (ENTREVISTADO 1, PESQUISA DIRETA, 2016). "No momento, temos que acelerar o projeto de adaptações dos apartamentos, esse é o grande objetivo da gestão do empreendimento" (ENTREVISTADO 2, PESQUISA DIRETA, 2016). 
Os meios de hospedagem devem atentar que algumas ações simples fazem toda a diferença no acolhimento de uma pessoa com deficiência ou mobilidade reduzida, mas nenhum dos entrevistados contemplou em sua colocação. Quanto ao fator ambiental, pode-se citar um balcão rebaixado na recepção para realizar o check-in (registro de entrada no meio de hospedagem) ou check-out (registro de saída do cliente do empreendimento) de um hóspede cadeirante, contemplando o aspecto atitudinal, encaminhar as bagagens ao apartamento do cliente.

Todas as colocações dos entrevistados limitam-se à deficiência física, motora. Não há menção às deficiências visuais e auditivas, por exemplo. Nesse contexto, cita-se como uma ação atitudinal, a necessidade de treinamentos para os funcionários do âmbito hoteleiro para auxiliar clientes dentro destes perfis, para que possam conduzir o acolhimento de forma abrangente e segura.

A quinta e última indagação analisou se o meio de hospedagem tinha o conhecimento sobre a legislação existente referente à acessibilidade na hotelaria. Constatou-se que um dos gestores têm ciência da lei, mas que não possui conhecimentos sobre a mesma, e que o outro gestor conhece bem as regras e exigências da área, como pode ser verificado nas falas abaixo.

"O que sabemos é que a acessibilidade se tornou uma obrigação, o ambiente deve estar adaptado e adequado ao cliente com deficiência, mas o aprofundamento e as cláusulas da lei, não conhecemos bem" (ENTREVISTADO 1, PESQUISA DIRETA, 2016). "Com certeza, nós temos consciência da legislação, a portamos no empreendimento, participamos inclusive de palestras a respeito" (ENTREVISTADO 2, PESQUISA DIRETA, 2016).

Os participantes da pesquisa sabem da existência da lei vigente no país, mas superficialmente, pois o gestor do meio de hospedagem que afirma conhecer bem a lei, ainda não adotou nenhuma iniciativa para tornar seu empreendimento acessível, tornando-se contraditório.

Cardoso e Strassburger (2012) atestam que na tentativa de atender às legislações, a grande maioria dos meios de hospedagem reserva uma pequena parte do número de apartamentos para portadores de mobilidade reduzida, colocando algumas barras de apoio no banheiro, acreditando assim, ter tornado o quarto acessível. Todavia, é sabido que outras condições são necessárias para adaptar um empreendimento hoteleiro adequadamente. Os mesmos autores alertam que "a hotelaria deve-se lembrar, e seguir, das demais exigências normativas das edificações, como por exemplo, rampas para acesso de pessoas usuárias de cadeira de rodas, elevadores mais largos, balcões de atendimento rebaixados, entre outras" (CARDOSO; STRASSBURGER. 2012, p. 14).

A conscientização e a mobilização dos gestores em adaptar seus meios de hospedagem, além de treinamento e qualificação de seus colaboradores, acarretarão em melhorias significativas para hóspedes com deficiência ou mobilidade reduzida, garantindo satisfação e total autonomia na estada dos mesmos nos meios de hospedagem.

\section{CONSIDERAÇÕES FINAIS}

O estudo analisou a hotelaria e acessibilidade no que tange a hospitalidade às pessoas com deficiência nos meios de hospedagem de Limoeiro do Norte-Ceará. A pesquisa teve caráter inovador, pois o estudo abordando a acessibilidade na hotelaria da cidade em questão era ausente.

O objetivo geral do estudo foi atingido, uma vez que foi analisada a acessibilidade dos meios de hospedagem da cidade em questão, podendo-se perceber que os empreendimentos hoteleiros ainda estão caminhando para uma hotelaria mais acessível.

O objetivo específico referente a acessibilidade dos meios de hospedagem para receber hóspedes com deficiência ou mobilidade reduzida e se esta poderia ser um diferencial nos empreendimentos foi atingido, pois foi detectado que um deles já buscou fazer algumas adaptações estruturais, porém não foram identificados outros serviços personalizados, direcionados a esse público. Enquanto o outro ainda está em processo de planejamento, ou seja, inexiste acessibilidade.

Quanto ao diferencial que a acessibilidade pode dar aos empreendimentos, estes focaram nas questões mercadológicas, de imagem do empreendimento. Porém, é de suma importância que a acessibilidade seja vista como uma possibilidade de inclusão e de abrangência dos mais diversos públicos, garantindo satisfação, conforto e segurança também das pessoas com deficiência ou mobilidade reduzida.

A identificação da demanda deste público nos meios de hospedagem analisados, também logrou êxito, pois identificou-se que a procura de pessoas com deficiência ou mobilidade reduzida nos empreendimentos ainda é incipiente, mas existe, o que requer dos gestores estratégias e adaptações físicas, conceituais e atitudinais para bem receber este público.

Ao buscar reconhecer possíveis melhorias no atendimento destes negócios para pessoas com deficiência ou mobilidade reduzida, obteve-se que os empreendimentos associam a hospitalidade à infraestrutura para 
este público. Mas como apontado ao longo do texto, este é o primeiro passo para romper as demais barreiras que estas pessoas enfrentam, rompendo os limites físicos, a convivência será potencializada e os outros entraves serão minimizados aos poucos.

O último objetivo específico visava verificar se os gestores possuem ciência da legislação sobre acessibilidade vigente no país, este foi atingido, uma vez que foi observado que os mesmos conhecem superficialmente a lei. Um dos entrevistados, inclusive, mencionou a aproximação com as normas, mas nada fez em seu empreendimento, o que é preocupante, afinal a acessibilidade necessita sair dos conceitos e partir para a prática.

Como pôde ser constatado neste estudo, a acessibilidade na hotelaria na cidade de Limoeiro do NorteCeará, ainda é um tema a ser explorado pelos gestores dos empreendimentos e espera-se que esta pesquisa possa expor a importância e a conscientização na adaptação dos meios de hospedagem para bem receber as pessoas com mobilidade reduzida ou com deficiência.

A fim de melhor atender este público, os meios de hospedagem devem se adaptar à legislação vigente no país, propor uma melhor qualificação de seus colaboradores na prestação de serviços, oferecer serviços personalizados a este público, dentre outros.

Esta pesquisa aponta outras possibilidades de estudo referente a esta temática: a acessibilidade nos meios de hospedagem na perspectiva do hóspede, os desafios do turismo acessível, a importância de uma sociedade inclusiva.

\section{REFERÊNCIAS}

ABNT. Associação brasileira de normas técnicas. acessibilidade de pessoas portadoras de deficiências a edificações, espaço, mobiliário e equipamentos urbanos: Nbr 9050/2015. ABNT: Rio de Janeiro. 2015.

\section{AGUIAR, M. F. Guia para elaboração do trabalho} de conclusão de curso. 2011. Disponível em: $<$ http://virtual-novo.ifce.edu.br> Acesso em: 04 mai. 2016.

BRASIL. Convenção sobre os Direitos das Pessoas com Deficiências. 2009. Disponível em: <http://www. pessoacomdeficiencia.gov.br/app/sites/default/files/ publicacoes/convencaopessoascomdeficiencia.pdf> Acesso em: 11 nov. 2018.

Decreto N 6949. Convenção Internacional sobre os Direitos das Pessoas com Deficiência. 2009. Disponível em: <http://www.turismo.gov.br/ acessibilidade.html> Acesso em: 03 mai. 2016.
Lei Brasileira de Inclusão da Pessoa com Deficiência (Estatuto da Pessoa com Deficiência). 2015. Disponível em: <http://www.planalto.gov. br/ccivil_03/_ato2015-2018/2015/lei/113146.htm> Acesso em: 11 nov. 2018.

CARDOSO, A. T.; STRASSBURGER, N. C. $A$ acessibilidade na hotelaria de Bento

Gonçalves - RS. 2012. Disponível em: <http: //www.ucs.br/ucs/tplVSeminTur\%20/eventos/ seminarios_semintur/semin_tur_7/gt08/arquivos/08/ 01_18_39_Cardoso_Strassburger>. Acesso em: 03 mai. 2016.

CARLETTO, A. C.; CAMBIAGHI, S. Desenho universal: um conceito para todos. São Paulo. 2008.

LASHLEY, C. Para um entendimento teórico. In: LASHLEY, C.; MORRISON, A. (Ed.). Em busca da hospitalidade: perspectives para um mundo globalizado. 1. ed. Barueri, SP: Manole, 2004.

MTUR. Acessibilidade. 2013. Ministério do turismo. Disponível em: <http://www.turismo.gov.br/ acessibilidade.html> Acesso em: 03 mai. 2016.

OLIVEIRA, L. M. B. Cartilha do Censo 2010 para pessoas com deficiência. 2012. Disponível em: <http://www.pessoacomdeficiencia.gov.br> Acesso em: 09 mai. 2016

SANSIVEIRO, S.; DIAS, C. M. M. Hotelaria

e Acessibilidade. 2005. Disponível em: <http://www2.anhembi.br/publique/media/simone>

Acesso em: 03 mai. 2016.

SASSAKI, R. K. O Conceito de Acessibilidade. 2006. Disponível em: <http://www.bengalalegal.com/ romeusassaki> Acesso em: 03 mai. 2016.

SILVA, A. C. L. Acessibilidade as pessoas portadoras de deficiência aos hoteis de Brasília 2004. Disponível em: <http://bdm.unb.br/bitstream/ 10483/263/3/2004_AnaCristinaLopesSilva.pdf>.

Acesso em: 11 nov. 2018.

SILVA, J. O. Modelo de Artigo, Timesharing como Estratégia de Marketing nos Meios de Hospedagem. 2015. Disponível em: $<$ http://virtual-novo.ifce.edu.br $>$ Acesso em: 09 mai. 2016.

STOLL, S. M. Hospitalidade: Conceitos e Reflexões Sobre sua percepção pratica dentre os hoteleiros do destino turística 
Balneário Camboriú - SC. 2006. Disponível em: <http://siaibib01.univali.br/pdf/Sueli\%20Stoll.pdf> Acesso em: 09 mai. 2016. 